\title{
Ectopic Gestation in Spleen: A Rare Case Presentation
}

\author{
Amruta Shrirang Padhye, Reeta Dhar and Shilpi Sahu
}

Department of Pathology, MGM Medical College, Kamothe, Navi Mumbai, India

\section{Dear Sir,}

We wish to bring to your attention a very rare site of ectopic gestation- the spleen. Ectopic pregnancy is defined as implantation of a fertilized ovum anywhere other than within the uterine cavity and occurs with an estimated incidence of $1.3-2.4 \%{ }^{[1]}$ The fallopian tube is the most common site for ectopic implantation which accounts for $95.5 \%$ of all ectopic gestations. ${ }^{[2]}$. Primary abdominal pregnancies have been described in a variety of extrapelvic organs, including omentum, liver, and small and large intestine. Primary splenic pregnancy is the least common site of extrauterine gestation with only 16 cases described until date. ${ }^{[3]}$

Here, we report a case of a 19 year old primigravida who came with complaints of pain in abdomen since 3-4 days with positive urine pregnancy test (UPT). Her ultrasonography (USG) was suggestive of moderate to severe hemoperitoneum with normal bilateral fallopian tubes and ovary and no evidence of intrauterine gestational sac. Beta HCG values were $>10,000 \mathrm{mIU} / \mathrm{ml}$. Exploratory laparotomy was done, hemoperitoneum noted (upto 800ml) and bleeding was seen on the superior surface of the spleen. Uterus, bilateral fallopian tubes and ovaries were normal with no evidence of any adnexal mass or ectopic tissue.

Dilatation and curettage was done and endometrial tissue was sent for histopathological examination which showed absence of trophoblastic tissue. Sections from splenic surface revealed presence of trophoblastic tissue (chorionic villi and decidua) along with splenic red pulp and hemorrhage. Abdominal pregnancies are defined as those occurring within the peritoneal cavity (excluding tubal, ovarian, or intraligamentous pregnancies) and account for $1.3 \%$ of ectopic pregnancies. They are classified as either primary or secondary; the latter is much more common. Primary abdominal pregnancies, which arise from fertilization of an ovum within the peritoneal cavity, are extremely rare. Although the most common sites of primary peritoneal pregnancy are the pouch of Douglas and the posterior uterine wall, primary implantation sites have been described in extrapelvic structures, including small and large intestine, omentum, liver and spleen.

USG and CT are very important to rule out ectopic pregnancy. Exploratory laparotomy should be done with precision taking care not to miss any ectopic pregnancy. However, supportive and confirmatory diagnosis is given by histopathology. In case of young females, presenting with acute abdomen, diagnosed as ectopic on ultrasonography/ laparoscopic examination, a thorough search for gestational sac or trophoblastic tissue should be done in rare sites like the peritoneal cavity and spleen in addition to the common sites like the fallopian tubes, ovary and broad ligaments.

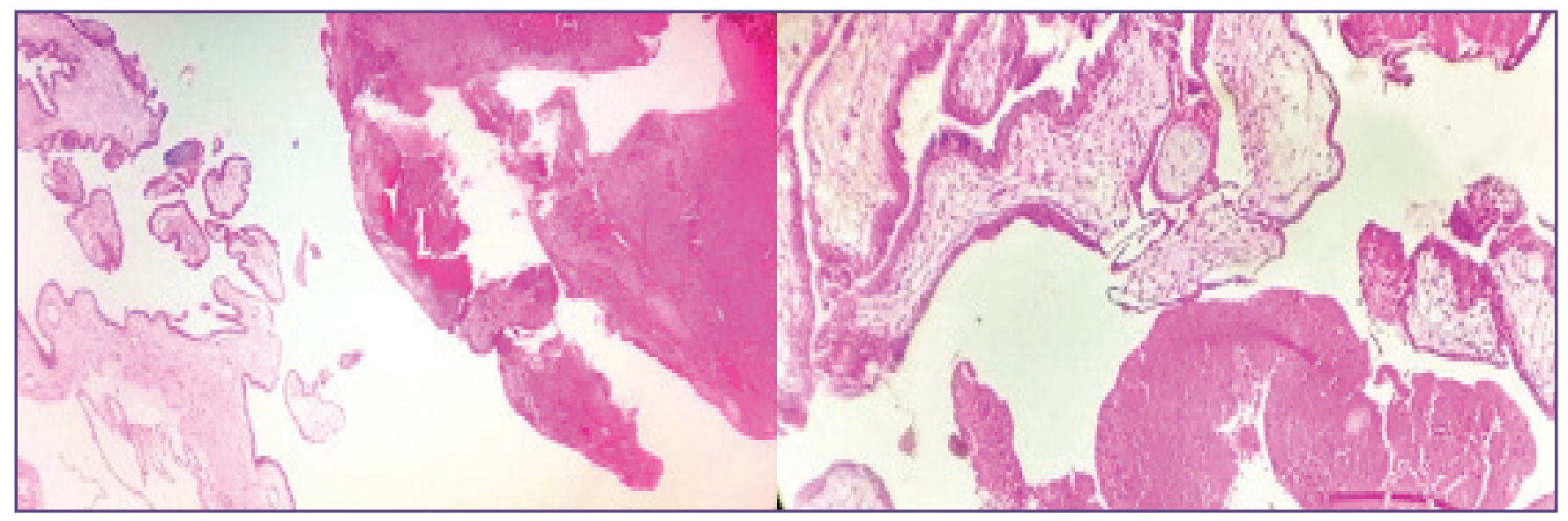

Fig. 1a \&1b: Trophoblastic tissue along with red pulp and hemorrhage. (H\&E - 40x). 


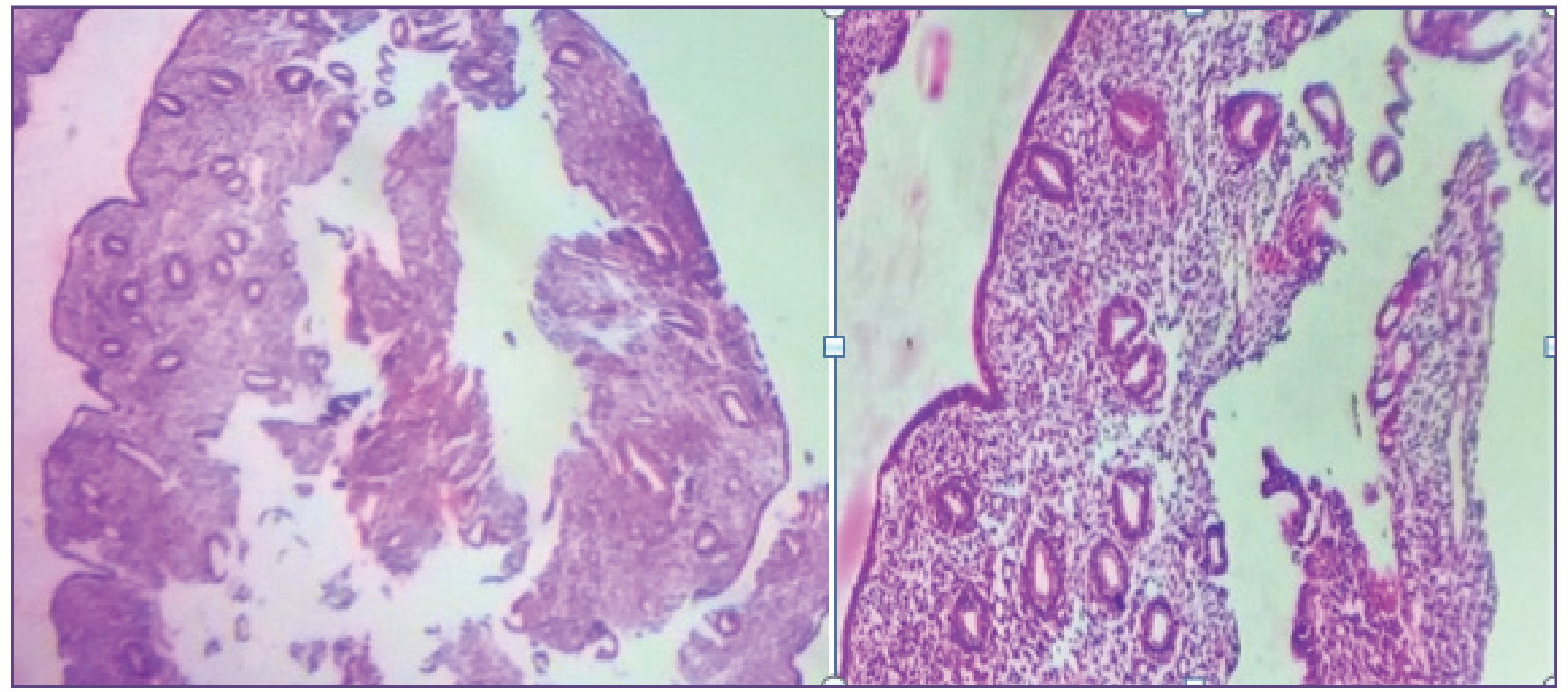

Fig. 2a \& 2b : Endometrial tissue, Absence of trophoblastic tissue. (H\&E-40x).

\section{References}

1. Taran FA, Kagan KO, Hübner M, Hoopmann M, Wallwiener D, Brucker S.. The Diagnosis and Treatment of Ectopic Pregnancy. Deutsches Ärzteblatt International. 2015;112(41):693-704.
2. Shaikh S., Jampala S., Devi S., Malika M. A Study of Ectopic pregnancy in a Tertiary Care Teaching Hospital. Indian Journal of Obstetrics and Gynecology Research 2016;3(2):132-136

3. Klang, E., Keddel, N., Inbar, Y. et al. Cardiovasc Intervent Radiol 2016;39: 1339. doi:10.1007/s00270-016-1403-5.

*Corresponding author:

Dr. Amruta Shrirang Padhye, 17/18, Radha Blocks, Shastri Hall, Javaji Dadaji Road, Grant Road (West), Mumbai 400007. India

Phone: $+919920950025,8422029680$

Email: amrutapadhye26@gmail.com

Financial or other Competing Interests: None.

Date of Submission : 02.11.2016

Date of Acceptance : 29.11.2016

Date of Publication : 19.02.2017 